\title{
Ein neuer Assurbanipal-Text.
}

\author{
Von Arthur Ungnad.
}

Im ersten Bande der Miscellaneous Inscriptions in the Yale Babylonian Collection (New Haven 1915) hat A. T. CLAY einen fragmentarischen Assurbanipal-Text in Urschrift vorgelegt, der die Bauten des Königs am Tempel der Ištar von Uruk berichtet. Es ist wohl eins der bekannten Tonfäßchen und deckt sich mehrfach mit dieser Textgattung im Wortlaut. ${ }^{\mathrm{T}}$ ) Die leider nicht gut erhaltene Einleitung bringt aber allerlei Neues. Geschrieben ist dieser neubabylonische Text vor 652, wie die Erwähnung des Šamaš-šum-ukîn beweist. Ein weiterer unedierter Bautext aus Uruk (Suppl. ; zu Bezold's Catal. Nr. 3333), auf den Streck, a. a. O., :S. CCCLXXXV verweist, ist mir unzugänglich. Der Text lautet:

"[a-na iluinnanna] urukki e-tel-lat samêe u irșitimtim iga-ri $\left.[t-t] i(\jmath)^{2}\right)$ ilânimes $s[i-i r-t i]^{2}[\ldots b] a(\jmath)-s i-l a[\ldots \ldots]$ ، la(l) kan-si [. .....] ${ }^{3}$ [la ma-gi-]ri iz-zi-ti sa ina ki-rib 'te-s[e-]e a(!)-sam-sa-nis $i-s u-u[r-r u]{ }^{4}$ [a-li-k]at $i$-di sarri mì-gior-i-sa mu-ra-si-[bat] ga-ri-[e-su] ${ }^{5}$ [be-li]t(i) mâtâti bami-mat par-si mus-te-si-rat gi-mir sú-lub-bu ${ }^{6}[a]-s i-b a t ~ \hat{e}-n e r-$ - gabl-iluz-nimn $\left.{ }^{3}\right)$ sa ki-rib $\hat{e}$-an-na be-el-ti rabî-ti bêlti(=:GAŠ $\left.A N\right)$ - su 7 Iinuasur-bân-apli sarru rabû sarru dan-nu sàr kissati sàr inât dis-sur sàr kib-rat irbi-ti šàr sarrânimes rubî̀ la sa-na-

I) Vgl. Streck's Assurbanipal S. 226 ff.

2) Vgl. den Asarhaddon-Text bei Cr.AY, Nr. 4I, I .

3) Nr. 41 : ê-ner-gal-an-na.

Zoitscir. f. Assyriologie, XXXI. 
an ${ }^{8}$ sa ul-tu tam-tim e-lit a-di tam-tion sap-lit i-bi-lu-ma gi-mir ma-lik(!) ú-sak-nis se-pu-us-su 9 sa e-li âlușur-ru sa ka-bal tam-tim e-lit u tilmunki sa ka-bal tam-tim sap-lit ${ }^{10} n i-r i$ be-lu-ti-su ú-kin-nu-úl-ma i-sú-țu ab-sa-an-su sa ip(!)se-tu-ú-su e-li kal ilânimes ta-a-ba(!) "' e-li șal-mat kakkkadi $d u-u s_{-}-s^{\prime} u-p a-t u$ rều-ú-su(!) sa ina palê-su iluadad zunnêmessu iluê-a nak̂-bi-su ${ }^{12}$ a-na ma-ti-su us-tab-ru-ú ina tub-du $u$ meś-ri-e is-te-ni-'-ú nisêmes-šu ${ }^{13}$ gi-mir um-ma-ni(!)-šu

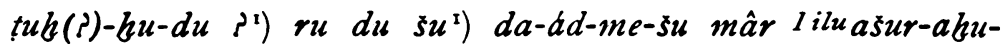
iddinana sarru rab̂̉ sarru dan-nu ${ }^{14}$ sàr kišsati sàr mât dssur sakkanak TIN.TIR.KI sàr mât sûmeri u akkadîki muse-sib TIN.TIR. KI e-pis $\hat{e}$-sag-ila ${ }^{15} m u$-ud-dis es-ri-e-te kul-lat ma-ba-zi sa ina kir-bi-si-na is-tak-ka-nu si-ma-a-ti ${ }^{16}$ mâr mâr Iilusin-ahbêmes-(e)rîba sarru rabû sarru dan-nu

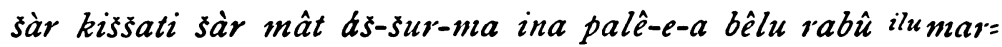
duk ${ }^{17}$ ina ri-sa-a-ti a-na TIN.TIR.KI i[-ru-um-ma sat-tukki $\hat{e}$-sag-ila] u ilânimes T[IN.T]IR.K[I ú-k[in] ${ }^{18}$ ki-din-nutu TIN.TIR.KI ak-șur ás-s[ú dan-nu ana ensi la ba-ba-li I ilu GIŠ.ŠIR-sumu-GI.NA] ${ }^{19} a-b u$ ta-li-me a-na sarru-ú-tu [bâb-iliki ap-ki-id u si-pir $\hat{e}$-sag-ila la ka-ta-a] ${ }^{20} \dot{u}$-sak-lil ina kaspi burâsi u ni-si[k-ti abni] mes $\hat{e}$-sag-i[la az-nun-ma $k i-m a$ si-țir bu-ru-mu] ${ }^{21} \dot{u}$-nam-mir $\hat{e}-s \grave{u}-a$ ù $s a$ es-ri-e-te $k a-l i-s\left[i-n a\right.$ bi-bi-il-ta-si-na ú-sal-lim] ${ }^{22}$ e-li kul-lat ma-bazi ú-sat(!)-ri-si an-dul-lum ina $\hat{u} m e[m e-s u-m a ~ \hat{e}-n e r-g a l-$ ilua-nim bît pa-pa-hi iluinnanna bêlti-ia $]^{2}{ }^{23}$ sa $l[a-b] a-r i s$ $i l-l i k-u-m a$ i-ku-pu ik-kam-ru') as-ra-t[i-su as-ti-'e-ma es-

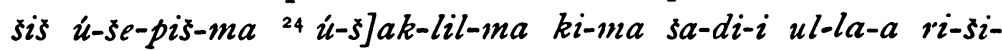
š́u-un a-n[a sat-ti iluinnanna urukki bêltiti rabî-ti 2.5 si]-pir sa-a-su ha-dis lip-pa-lis-ma ja-a-ti I iluasur-bân-apli sàr onât $a \hat{s}-s u\left[r\right.$ rubê pa-lib-sa a-mat saldamikti-ja] ${ }^{26}$ lis-sa-kin sap-

I) Spuren von $i$ ? Die Deutung dieser 3-4 Zeichen ist mir unklar; im Parallelismus zu tuhbudu erwartet man eine ähnliche Form, aber weder ru-dusu noch $s u b-d u-s u$ (S) sieht vertrauenerweckend aus. Außerdem inüßte das Zeichen vor $r u$ unberücksichtigt bleiben.

2) So vielleicht nach Nr. 4I, I I zu ergänzen.

3) Sonst steht an dieser Stelle in-nab-tu ( $\left.\mathrm{L}^{2} 17 ; \mathrm{L}^{6} 18\right)$. 
tu-us-sa balât ûmemes arkîtimes se-bi-e lit-tu-[tu li-sim sima-ti] ${ }^{27} \grave{u}(!)$ sa IiluGIŠ.ŠIR-šumu-GI.NA sàr bâb-íliki $a-b u$ ta-lim-[ja ûmeme-su li-ri-ku lis-bi bu-'-a-ri] ${ }^{28}$ a-a-um-ma

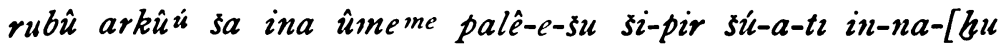
all-bu-us-su lu-ud-dis $\left.{ }^{29}{ }^{9} u ́-m e\right]$ it-ti sumi-suu lis-țr musarûua li-mur-ma samnu lip-šu-[us immer nik̂u lik-ki $\left.{ }^{\circ} i t-t\right] i$ mu= sarêe-su liš-kun ik-ri-bi-su iluinnanna urukki $i-s e m-m e ~ s a$ sú-[me sat-ru ${ }^{3}$ ì sum ta-lim-j]a i-a-ab-ba-tu lu-u a-sar-su ú-nak-ka-ru-ma it-ti musa[rêe-su la i-sak-ka-nu ${ }^{2}$ iluinnanna uruk]ki bêlti rabi-ti ag-gis lik-kil-me-šu-ma sum-su zêr-su ina mâtâti li-bal-lik.

Übersetzung.

- [Der Innanna] von Uruk, der Herrscherin von Himmel und Erde, der Starken(?) unter den Göttern, der [Erhabenen], ${ }^{2}[\ldots . .$. . . . , [die ... .] die nicht Unterwürfigen(?), [die ..... ${ }^{3}$ die Unbotmäfigen, der Grimmen, die mitten im Wirrsal gleich dem Wirbelsturm einhertanzt, ${ }^{1}$ ) 4 die dem Könige, ihrem Liebling, zur Seite [geht] und [seine] Feinde niederschlägt, ${ }^{5}$ [der Herrin](?) der Länder, die die (göttlichen) Gebote entscheidet, die alle Satzungen richtig lenkt, ${ }^{6}$ die in Energalanim in Eanna wohnt, der großen Herrin, seiner Herrin, -

7 Assurbanipal, der große König, der mächtige König, der König der Welt, der König von Assyrien, der König der vier Weltteile, der König der Könige, der unvergleichliche Fürst, ${ }^{8}$ der (das Gebiet) vom oberen Meere bis zum unteren Meere als Eigentum nahm und alle Fürsten seinem Fuße unterwarf, ${ }^{9}$ der auf Tyrus mitten im oberen Meere und Tilmun mitten im unteren Meere ${ }^{10}$ das Joch seiner Herrschaft festlegte, sodaß sie sein Geschirr $^{2}$ ) zogen, dessen

I) Zu sâru vgl. auch noch UM V I 49, 19: $U L . U L=$ sa-a-ru-um; ebens.o 136, Rs. V 7. (Bei Delitzsch, Hwb. bekanntlich zum Teil noch unter רוש.) Die Bedeutung ist «springen, tanzen".

2) abłânu eigentlich wohl = nîru; vgl. auch STREck, Assurb. S. 430 . 
Taten allen Göttern wohlgefällig, "I dessen Hirtentum den Schwarzköpfigen gar angenehm war, zu dessen Regierung Adad seine Regen- und Ea seine Quellwasser ${ }^{12}$ seinem Lande in Fülle spendeten, (der) in Überfluß und Reichtum seine Leute weidete, ${ }^{\mathrm{I}}{ }^{\mathrm{r}}{ }^{\mathrm{3}}$ (sodaß) sein ganzes Volk üppig reich(?) und seine Wohnstätten blühend(?) waren;

der Sohn Asarhaddon's, des großen Königs, des mächtigen Königs, ${ }^{14}$ des Königs der Welt, des Königs von Assyrien, des Statthalters von Babylon, des Königs von Sumer und Akkad, der Babylon (neu) besiedelte und Esagila herstellte, ${ }^{15}$ der die Heiligtümer aller Städte erneuerte, der in ihnen die (nötige) Ausstattung anbrachte;

${ }^{16}$ der Enkel Sanherib's, des großen Königs, des mächtigen Königs, des Königs der Welt, des Königs von Assyrien.

Während meiner Regierung zog der große Herr Marduk ${ }^{17}$ unter Jubel in Babylon [ein, worauf ich die festen Abgaben für Esagila] und die Götter von Babylon bestimmte. ${ }^{18}$ Die Privilegien(?) Babylon's setzte ich (neu) fest. ${ }^{2}$ ) Damit [der Starke den Schwachen nicht unterdrücke], ${ }^{19}$ bestellte ich meinen gleichberechtigten Bruder [Šamaš-šum-ukîn] zur Königsherrschaft [über Babylon, und die unfertige Arbeit an Esagila] ${ }^{20}$ vollendete ich. Mit Silber, Gold und Edel[steinen stattete ich] Esagila [aus, und wie die Schrift des Firmaments] ${ }^{2 x}$ ließ ich Ešua erstrahlen. Auch aller (übrigen) Heiligtümer [Beschädigung machte ich wieder gut]. ${ }^{22}$ Über alle Städte breitete ich Schutz aus.

Damals habe ich, [was Energalanim, das Heiligtum der Ištar, meiner Herrin, betrifft], ${ }^{23}$ das alt geworden, verfallen und zerstört war, um dessen (heilige) Stätten [mich gekümmert, (sie) neu herstellen lassen und] ${ }^{24}$ vollendet; gleich einem Berge habe ich sie hoch aufgeführt.

Für [immerdar(?) $\left.{ }^{3}\right)$ möge Ištar von Uruk, meine große Herrin], ${ }^{25}$ jenes Werk freudig anschauen und für mich,
1) isteni' $u=$ irteni' $u$.
2) Wörtlich *band ich».
3) Oder «deshalb»? 
Assurbanipal, den König von Assyrien, [den Fürsten, der sie verehrt, gute Worte] ${ }^{26}$ auf ihren Lippen haben. Leben langer Tage, Sättigung an Nachkommenschaft [möge sie mir zum Geschenk bestimmen]. ${ }^{27}$ Auch des Šamaš-šumukîn, des Königs von Babylon, meines gleichberechtigten Bruders, [Tage mögen lange währen, er möge sich sättigen an Glück].

${ }^{28}$ Irgend welcher spätere Fürst, während dessen Regierungszeit dieses Werk baufällig [wird, möge seinen Verfall erneuern! ${ }^{29}$ Meinen Namen] möge er mit seinem Namen hinschreiben, meine Inschrift möge er ansehen und mit Öl salb[en, ein Opfer möge er spenden und (sie) $\left.3^{\circ} \mathrm{mit}\right]$ seiner Inschrift anbringen: seine Gebete wird Ištar von Uruk erhören! Wer meine [Namensschrift ${ }^{3^{\mathrm{x}}}$ und den Namen meines gleichberechtigten (Bruders)] zerstört oder ihre Stelle ändert und sie nicht mit [seiner] Inschrift [anbringt,] den möge $3^{32}$ [Ištar von Uruk], (meine) große Herrin, zornig anblicken und seinen Namen und Samen in den Landen vernichten! 